CHAPTER

\title{
Correlative Cryo-
} Fluorescence

Light Microscopy and

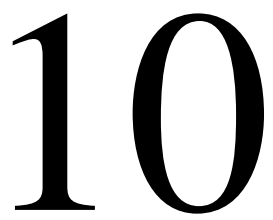

Cryo-Electron Tomography of Streptomyces

Roman I. Koning ${ }^{*}$, Katherine Celler ${ }^{\dagger}$, Joost Willemse ${ }^{\dagger}$, Erik Bos*, Gilles P. van Wezel ${ }^{\dagger}$, Abraham J. Koster*

* Department of Molecular Cell Biology, Section Electron Microscopy, Leiden University Medical Center, Leiden, The Netherlands

${ }^{\dagger}$ Molecular Biotechnology, Institute of Biology Leiden, Leiden University, PO Box 9505 ,

\section{CHAPTER OUTLINE}

Introduction.

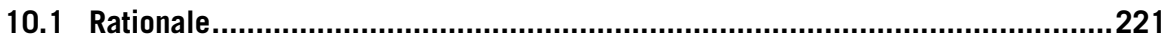



10.2.1 Cell Culturing ............................................................ 222

10.2.2 Fluorescent Labeling and Fiducial Marker Addition................... 222

10.2 .3 Cryo-Fixation.......................................................... 223

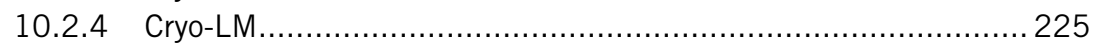

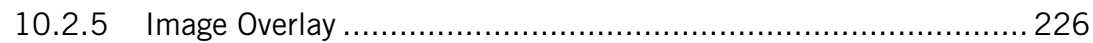

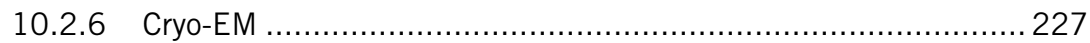

10.2.7 Cryo-Electron Tomography ............................................. 228

10.2.8 Tomographic Reconstruction .............................................. 229

10.2.9 Segmentation and Volume Rendering ................................... 230

10.2.10 Visualization of Correlative Data...................................... 232

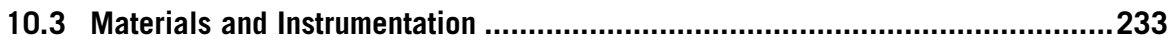

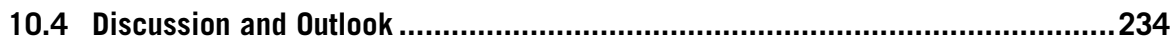

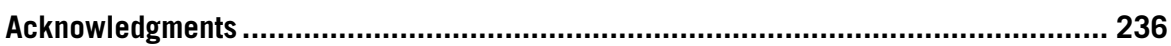

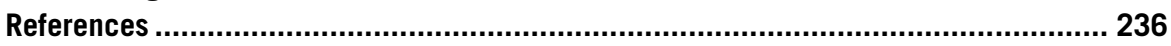




\begin{abstract}
Light microscopy and electron microscopy are complementary techniques that in a correlative approach enable identification and targeting of fluorescently labeled structures in situ for three-dimensional imaging at nanometer resolution. Correlative imaging allows electron microscopic images to be positioned in a broader temporal and spatial context. We employed cryo-correlative light and electron microscopy (cryo-CLEM), combining cryo-fluorescence light microscopy and cryo-electron tomography, on vitrified Streptomyces bacteria to study cell division. Streptomycetes are mycelial bacteria that grow as long hyphae and reproduce via sporulation. On solid media, Streptomyces subsequently form distinct aerial mycelia where cell division leads to the formation of unigenomic spores which separate and disperse to form new colonies. In liquid media, only vegetative hyphae are present divided by noncell separating crosswalls. Their multicellular life style makes them exciting model systems for the study of bacterial development and cell division. Complex intracellular structures have been visualized with transmission electron microscopy. Here, we describe the methods for cryo-CLEM that we applied for studying Streptomyces. These methods include cell growth, fluorescent labeling, cryo-fixation by vitrification, cryo-light microscopy using a Linkam cryo-stage, image overlay and relocation, cryo-electron tomography using a Titan Krios, and tomographic reconstruction. Additionally, methods for segmentation, volume rendering, and visualization of the correlative data are described.
\end{abstract}

\title{
INTRODUCTION
}

Correlative light and electron microscopy (CLEM) is a combination of imaging techniques in which the same object is visualized using both light microscopy (LM) and electron microscopy (EM). CLEM is employed if a specific biological question cannot be answered by LM or EM alone and cellular morphology as well as functional, dynamic, or positional information of cellular structures is needed. In CLEM on biological samples, fluorescence microscopy (FM) is generally employed to target sparse, transient, or specifically labeled (protein) structures for structural imaging using EM.

The specific advantage of EM is that cell morphology and specific macromolecular structures can be imaged in three dimensions at nanometer scale resolution $\left(\sim 2 \times 10^{-9} \mathrm{~m}\right)$. EM provides images of fixed, static structures with a maximum thickness of several hundred nanometers and a field of view of several micrometers, often much smaller than one cell. Larger volumes can be imaged by serial sectioning and the field of view can be enhanced by combining images into montages (Faas et al., 2012). The advantage of LM is that large areas can be dynamically imaged using a variety of genetically encoded or chemical fluorescent labels. Imaging resolution is, however, two orders of magnitude lower $\left(\sim 1 \times 10^{-7} \mathrm{~m}\right)$ and does not provide detailed information about labeled or unlabeled structures.

The main difficulty of CLEM is related to compatibility of specimen preparation techniques. Standard (chemical) fixation techniques for LM generally do not optimally preserve the ultrastructural for EM. Conventional EM specimen techniques, which include dehydration, plastic embedding, and heavy metal staining, negatively 
affect the usability of fluorescent dyes, though useful protocols are emerging (Karreman et al., 2012; Kukulski et al., 2011). These disadvantages are not encountered when cryo-fixation (either plunge freezing or high-pressure freezing) is used in combination with cryo-electron microscopy (cryo-EM) (Le Gros, McDermott, Uchida, Knoechel, \& Larabell, 2009; Schwartz, Sarbash, Ataullakhanov, Mcintosh, \& Nicastro, 2007; van Driel, Valentijn, Valentijn, Koning, \& Koster, 2009). Vitrification is a cryo-fixation process in which the cooling rate is so high $\left(>100.000{ }^{\circ} \mathrm{C} / \mathrm{s}\right.$ for plunge freezing) that water does not crystallize, but instead adopts a glass-like structure that perfectly preserves biological samples (Dubochet et al., 1988).

Vitrification is essential for cryo-CLEM on cells. First, whole bacteria and parts of cells that are thinner than a few microns can be excellently preserved in a (close to) native state, allowing unperturbed and detailed three-dimensional high-resolution cryo-imaging of subcellular structures (Koning, 2010; Lucic, Leis, \& Baumeister, 2008; Pilhofer, Ladinsky, McDowall, \& Jensen, 2010). Second, most fluorescent dyes and labels that are employed for live cells seem to retain their fluorescence after vitrification while being kept at cryo-conditions. Vitrified cells can be imaged using both (phase contrast) cryo-EM, without using additional contrasting agents, and fluorescence with bright field LM. Samples that are thinner than half a micron can be imaged whole, without specimen thinning (by cryo-sectioning or focused ion beam milling) (Marko, Hsieh, Schalek, Frank, \& Mannella, 2007; Rigort et al., 2010). Streptomyces are 500-800 $\mathrm{nm}$ in thickness and are therefore suitable for visualization by whole-cell cryo-EM.

The major disadvantage of cryo-EM is that prolonged exposure to the electron beam increasingly damages the sample, as electrons are interacting strongly with vitrified biological samples. Therefore, searching must be performed fast, using a limited electron dose rate and at low magnification, resulting in noisy images and obscuring potential structures of interest. In addition, techniques to tag and identify specific proteins or molecules by labeling inside cells with electron dense labels are currently very limited (Diestra, Fontana, Guichard, Marco, \& Risco, 2009; Risco et al., 2012; Wang, Mercogliano, \& Lowe, 2011). Consequently, it is challenging to identify and target specific subcellular structures other than those which can be identified by their morphology, like membranes, the cytoskeleton, or ribosomes. FM offers advantages to cryo-EM with respect to labeling and targeting specific structures. Macromolecular structures inside cells can be fluorescently labeled using genetic fusion proteins like eGFP or using site-specific chemical labels. This allows searching relatively large areas for locations with labeled structures, while minimizing imaging using electrons.

Cryo-CLEM on thin samples is a relatively fast technique compared to many other CLEM techniques, as lengthy specimen preparation procedures for freeze substitution, preparation of markers, and sectioning are not needed. Fluorescent labeling, vitrification, cryo-fluorescence imaging transfer, and relocation in the transmission electron microscope (TEM) followed by cryo-ET can be carried out within 1 day. Numerous positions on one EM grid can be targeted in the light microscope after which tens of tomograms can be recorded in batch in a few days. 
In conclusion, cryo-CLEM, combining FM and cryo-electron tomography, surmounts the limitations of each individual technique with respect to resolution, scale, dynamics, and labeling. Cryo-CLEM allows live imaging of infrequent and/or transient events and targeting of labeled structures inside cells using LM, providing a broader historical and spatial context to high-resolution structural 3D maps obtained using EM.

Cryo-CLEM on whole cells is facilitated by their natural thickness. With an average diameter of a few microns, bacteria are generally one or two orders of magnitude smaller than eukaryotic cells. Some bacteria (including Streptomyces) even fall within the thickness limit for tomography $(\sim 500 \mathrm{~nm})$, making them particularly suitable for whole-cell cryo-CLEM without the need for sectioning (Morris \& Jensen, 2008). In addition, culturing and genetic manipulation is more straightforward in prokaryotic organisms, which provides an additional advantage. Additionally, correlation of LM and EM images of fluorescently labeled bacteria on an EM grid is easier, since the pattern in LM is comparable to the electron dense pattern in cryo-EM. This is even more apparent for filamentous bacteria like Streptomyces, which form specific thread-like patterns (Celler, Picioreanu, van Loosdrecht, \& van Wezel, 2012). These multicellular filamentous bacteria are commonly found in the soil (Claessen, Rozen, Kuipers, Sogaard-Andersen, \& van Wezel, 2014). Their complex life cycle starts with the germination of a spore, which grows out by apical extension and branching to form a dense network of hyphae that is referred to as vegetative (or substrate) mycelium (Chater \& Losick, 1997). Upon nutrient depletion, this mycelium is autolytically degraded to produce the nutrients for the next phase of the life cycle, whereby erected sporogenic hyphae are produced that form the aerial mycelium. This stage of the Streptomyces life cycle coincides with antibiotic production (Bibb, 2005; van Wezel \& McDowall, 2011). The aerial hyphae differentiate into chains of spores involving a spectacular process of coordinated cell division and DNA segregation and DNA segregation and condensation, whereby up to 100 uninucleoid spores are produced (Schwedock, McCormick, Angert, Nodwell, \& Losick, 1997).

Although the Streptomycetes are commercially widely used as producers of natural products (in particular antibiotics) and industrial enzymes (van Wezel, McKenzie, \& Nodwell, 2009), there is still much to be learned about the molecular and structural basis of growth, morphogenesis, and division. During the Streptomyces life cycle, two distinct forms of cell division occur (McCormick, 2009). During sporulation-specific cell division, a thick spore wall consisting of peptidoglycan is synthesized and remodeled during the metamorphosis of unicellular prespore compartments into mature spores (Jakimowicz \& van Wezel, 2012). In vegetative hyphae, crosswalls form at irregular intervals of roughly 5-10 $\mu \mathrm{m}$ (depending on the specific strain) to delimit multigenomic compartments. These compartments usually contain an irregular number of chromosomes and replication machinery in different stages of assembly and disassembly, sometimes within the same compartment. Unlike aerial septa, crosswalls do not constrict the cell and do not result in cell fission, effectively constituting a completely different form of cell division. Many of the 
divisome components which are required for septal peptidoglycan synthesis in canonical cell division such as found in Bacillus subtilis and Escherichia coli (Errington, Daniel, \& Scheffers, 2003), and during sporulation-specific cell division in Streptomyces, are not required for cross-wall formation (Mistry, Del Sol, Wright, Findlay, \& Dyson, 2008). These crosswalls delimit the syncytial cells, but do not constrict, and do not lead to cell fission. Live imaging of vegetative hyphae has demonstrated that membranes, enriched in phosphatidylglycerol and cardiolipin, dynamically localize during growth, ultimately localizing to the sites of cross-wall formation. In order to study these membranes in more detail, we developed cryoCLEM, combining cryo-electron tomographic structural studies with functional LM assays.

\subsection{RATIONALE}

We performed cryo-CLEM on Streptomyces (i) to investigate the localization of DNA and lipids vegetative hyphae, (ii) to develop a workflow for correlative fluorescence LM and cryo-electron tomography of vitrified whole cells, and (iii) to develop a workflow in which all correlative data, including (fluorescent) LM, cryo-EM, cryo-ET, and surface-rendered data, are visualized in a conceptual manner.

Streptomyces are suitable for the development of a cellular cryo-CLEM workflow for several reasons. (1) Streptomyces are thin enough $(500-800 \mathrm{~nm})$ for whole-cell cryo-fixation by plunge freezing, which ensures excellent preservation of structural detail. (2) The three-dimensional structure can be determined using cryo-ET along consecutive parts of whole, unsectioned, vegetative mycelium of Streptomyces, when liquid culture samples are deposited sparsely on an EM grid and vitrified. (3) Position relocation from LM to EM and correlation of recorded images can be performed without the use of additional markers or finder grids, because the mycelium has a unique and distinct morphology that are similar in both imaging modalities.

Cryo-EM is useful for CLEM research since vitrification preserves fluorescence of many chemical dyes and fluorescent proteins, which makes LM at cryogenic temperatures possible. Another advantage is that the cryo-CLEM technique described here is fast and can be performed within a day.

\subsection{METHODS}

Cryo-CLEM is described here in six general steps comprising two specimen preparation steps, two imaging steps, and two image processing steps (Fig. 10.1), which comprise the following practical sequence: cell culturing, fluorescent labeling and fiducial marker addition, cryo-fixation, cryo-LM, image overlay, relocation, cryo-EM, cryo-electron tomography, tomographic reconstruction, segmentation and volume rendering, and visualization of correlative data. 

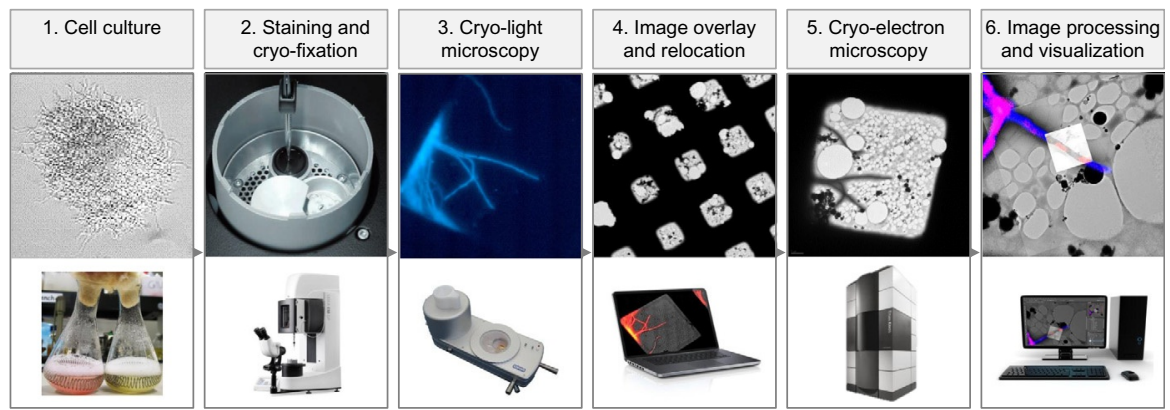

FIGURE 10.1

Schematic overview of the workflow used for the investigation of Streptomyces bacteria using cryo-CLEM. The complete workflow includes six steps. (1) Cells are cultured and fluorescently stained. (2) These cells are applied to an EM support grid, blotted, and vitrified using a plunge-freezing device. (3) The vitrified fluorescently stained cells on the EM grid are transferred to a cryo-stage and imaged at cryogenic conditions by cryo-FM. (4) The specimen is transferred into a TEM and the positions at which the cryo-FM images were acquired are visually relocated using the morphology of the Streptomyces mycelium. (5) Cryo-EM projection images at different magnifications are acquired for correlation and overlay of images. Cryo-ET is performed at targeted positions. (6) Visualization was performed by overlaying cryo-LM, cryo-EM, and 3D cryo-ET data using Photoshop, Amira, and Premiere.

\subsubsection{CELL CULTURING}

For plunge freezing, Streptomyces bacteria were grown in liquid culture, where they form a branched network of hyphae otherwise known as a vegetative mycelium.

1. Germinating spores. Spores $20 \mu \mathrm{L}$ of Streptomyces albus $\mathrm{J} 1074$ containing roughly $10^{7}-10^{9}$ spores $/ \mathrm{mL}$ were added to $20 \mathrm{~mL} 2 \times$ YT medium (Kieser, Bibb, et al., 2000) containing $10 \%$ sucrose.

2. Growing cells. The spore suspension was grown for $10-12 \mathrm{~h}$ at $30^{\circ} \mathrm{C}$ at $300 \mathrm{rpm}$ stirring speed.

\subsubsection{FLUORESCENT LABELING AND FIDUCIAL MARKER ADDITION}

Immediately prior to vitrification, cell cultures were washed, and dyes for fluorescence LM and fiducial nanogold for electron tomography were added.

1. Washing cell culture. $1 \mathrm{~mL}$ of cell culture was pipetted into an Eppendorf and $0.5 \mathrm{~mL}$ PBS buffer was added to wash the culture and remove sucrose. After mixing by inverting the Eppendorf, the culture was spun down gently at $1000 \mathrm{rpm}$, and $1 \mathrm{~mL}$ of supernatant was removed. The washing was repeated twice; in each case, $1 \mathrm{~mL}$ of PBS was added.

2. Adding fluorescent dyes. Depending on the structures to be labeled, different fluorescent dyes were added. Note that stains that were used are routinely used for 
live microscopy to minimize secondary effects on morphological and functional integrity. FM5-95 was used to stain anionic phospholipids, including phosphatidylglycerol. 10-Nonyl acridine orange (NAO) was used to preferentially stain cardiolipin. DAPI was used to stain DNA. Note that the dyes were chosen such that the excitation/emission spectra allowed dual labeling and combination of FM5-95 and DAPI, or NAO and DAPI. All dyes were obtained from Molecular Probes. Roughly $1-5 \mu \mathrm{L}$ of dye was added to a $40 \mu \mathrm{L}$ sample, depending on dye intensity.

3. Adding fiducial gold. Fiducial markers were added to the samples to aid tracking and alignment during tilt series acquisition and tomographic reconstruction. Final dilution varied with the concentration of the Au particles and the desired density of the fiducials on the grid. In general, a good fiducial density was obtained when to a $40 \mu \mathrm{L}$ sample, $1 \mu \mathrm{L}$ of $15 \mathrm{~nm}$ protein A-stabilized $\mathrm{Au}$ particles were added.

\subsubsection{CRYO-FIXATION}

1. Selecting EM grid and support film. In CLEM, finder grids and relocation markers are often used to target positions between LM and EM modes. Streptomyces vegetative mycelium formed a unique pattern when placed on an EM grid. This pattern was similar when imaged by transparent cryo-LM and low-magnification cryo-EM conditions. Therefore, targeted positions were easily recognized and correlated, and it was not necessary to use finder grids for relocation. In practice, a variety of different specimen support grids were used. In order to maintain support film stability and allow tilting for tomography, 150-300-mesh-sized grids were used. As specimen support film, generally Quantifoil or lacey carbon films were used. Though in cryo-EM on adherent cells inert gold grid were necessary to allow cell growth, in this case copper grids were used since the bacteria were only in contact with the grids for a limited amount of time $(<1 \mathrm{~min})$ prior to vitrification.

2. Glow-discharging the EM grid. To render the EM grids hydrophilic, grids were glow-discharged before use. They were placed onto a metal mesh with the carbon side facing up. Grids were glow-discharged for 1-2 min at $\sim 20 \mathrm{~mA}$ in $2 \times 10^{-1}$ mbar air. Grids were used within an hour after glow-discharging.

3. Preparing the cryo-fixation device. The plunge-freezing device (Leica EM GP) was switched on, the water container for the humidifier was filled with $60 \mathrm{~mL}$ distilled water, and filter paper was placed (Whatman no 4. or Schleicher and Schuell no. 595 or 597) into position inside the environmental chamber. The filter paper counter was reset to zero before starting. A cryo-storage box for vitrified grids was marked for identification of the sample throughout the experiment and positioned into the transfer container. The liquid nitrogen Dewar was filled with $(1.5 \mathrm{~L})$ liquid nitrogen. When the ethane container reached liquid nitrogen temperature, the (black) secondary cryogen container was filled with ethane or ethane/propane (Tivol, Briegel, \& Jensen, 2008) using the cryogen liquefier. 
4. Setting vitrification parameters. Using the control panel, the environmental chamber temperature was set to $22{ }^{\circ} \mathrm{C}$ and the humidity (Hr) to $95 \%$. The blotting time was set to $1-2 \mathrm{~s}$, blotting once from one side, with no waiting time after blotting. The liquid ethane temperature was set to $-181^{\circ} \mathrm{C}$ when liquid ethane was used, or to $-191{ }^{\circ} \mathrm{C}$ when a liquid ethane/propane mixture was used.

5. Sample vitrification. The sample $(3 \mu \mathrm{L})$ was applied to the carbon side of the glow-discharged grid. This was done outside the environmental chamber, since cell growth and blotting was performed at room temperature. The grid was clamped with a forceps such that the sample was blotted from the carbon facing side. The Leica EM GP rod was set in the "Load forceps" position and the tweezers were mounted on the forceps interlock. The automatic vitrification process was activated, initiating lowering of the environmental chamber, grid blotting, and plunge freezing.

6. Storage and transfer of vitrified grid. After plunging, the vitrified grid was transferred from the liquid ethane into a cryo-grid box for transfer and storage. At this point, if desired, more grids were vitrified (repeat step 5). The cryo-grid box was stored under liquid nitrogen in $50-\mathrm{mL}$ polystyrene tubes (Greiner, The Netherlands), which were placed in liquid nitrogen Dewars (Cryotech, The Netherlands). When the grids were directly used for cryo-LM, up to three grids were positioned in a Linkam transfer cassette that could be held in a transfer box (Fig. 10.2C).
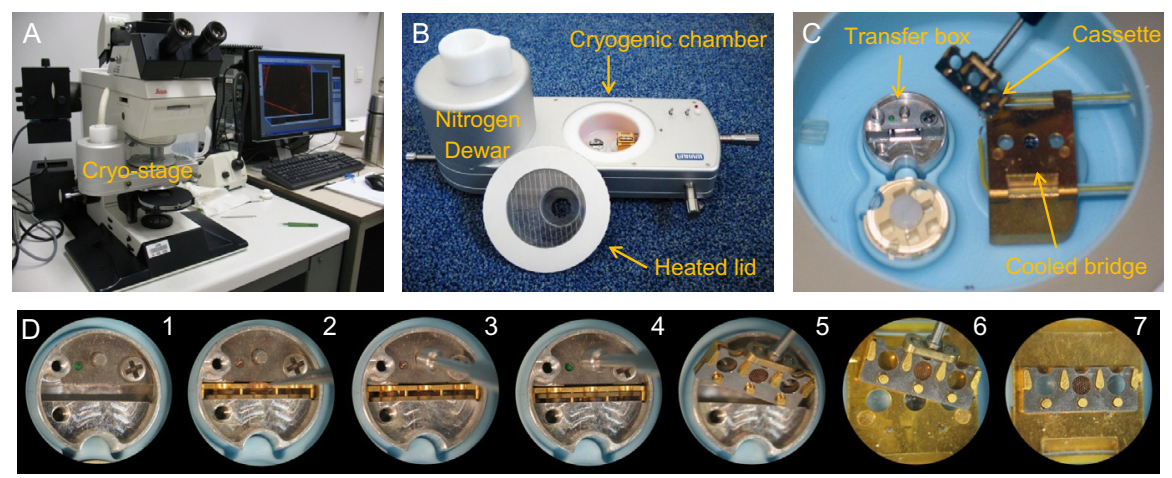

\section{FIGURE 10.2}

The Linkam correlative CMS196 cryo-LM stage. The cryo-LM setup consists of an upright light microscope under which the cryo-stage is mounted (A). The cryo-stage has a nitrogen Dewar and a cryogenic chamber for the samples, which is covered by a heated lid with a depressed opening in which the lens is positioned (B). In the cryogenic chamber, vitrified grids can be transferred from a cryo-grid box to the transfer box, which is used to transfer grids into a cassette onto the cooled bridge for imaging (C). The transfer box (D1) holds the cassette in which a EM grid is placed (D2) and a mechanism for closing the cassette (D3) so the EM gird is magnetically clamped inside (D4). Using a magnetic tool, the cassette can be picked up (D5) and transferred-at liquid cryogenic conditions - to the cooled bridge (D6 and C) at which it can be imaged (D7). 
More detailed descriptions and protocols for using the Leica EM GP or vitrobot plungers can be found elsewhere (Iancu et al., 2006; Resch, Brandstetter, Königsmaier, Urban, \& Pickl-Herk, 2011).

\subsubsection{CRYO-LM}

The Linkam correlative cryo-stage. For cryo-LM, a Linkam CMS196 correlative microscopy cryo-stage was used (Linkam Scientific Instruments, UK). This stage was specifically designed for light microscopic imaging of cryogenically cooled samples that are prepared on EM grids. The cryo-stage is suitable for upright light microscopes (Fig. 10.2A), in both fluorescence and bright field mode. The cryo-stage is equipped with its own liquid nitrogen Dewar that is coupled to a filling system, which automatically regulates the nitrogen levels in the cryogenic chamber (Fig. 10.2B). This chamber could be kept at cryogenic temperatures for about 30 min without refilling the nitrogen Dewar. The cryogenic chamber has a heated lid, which prevents frost contamination, with a depressed circular opening in which the lens is positioned (Fig. 10.2B). Manual sample movement in the horizontal plane was achieved by rotation of two screws on the cryo-stage, while focusing was performed by changing the height of the microscope stage on which it was mounted.

The cryo-stage is optimized for handling EM grids at cryogenic conditions while minimizing ice contamination from air and specimen warming. The stage includes a specific grid transfer system that comprises a cassette and a transfer box (Fig. 10.2C and D). The cassette holds up to three grids and is made up of two metal sheets with three holes that are clamped together with small magnets (Fig. 10.2D, 2-7). The transfer box holds one cassette for transfer, but also contains a mechanism to open and close the two magnetically clamped sheets of the cassette, allowing vitrified grids to be loaded into the cassette (Fig. 10.2C and D, 1-5). The cassette, with vitrified grids, can be swiftly moved with a special magnetic rod from the transfer box to a cooled metal bridge (Fig. 10.2C and D, 5-6). There the cassette is, again magnetically, clamped to the cooled bridge where it can be imaged (Fig. 10.2B and C). The Linkam cryo-stage has designated positions for two cryo boxes to facilitate transfer of grids that are stored in standard cryo boxes into the Linkam transfer box.

1. Preparing the light microscope. The light microscope and the lamp for bright field microscopy were activated. The postmagnification lens was set to $1.0 \times$ and the $100 \times$ objective lens (Fluortar $100 \times$ dry objective lens with a numerical aperture (NA) of 0.71 and a working distance of $4.7 \mathrm{~mm}$ was selected). The camera control and image acquisition computer and the Leica AF Lite software were started.

2. Preparing the cryo-stage. The cryo-stage was switched on, the Dewar was filled with liquid nitrogen, and the automated filling system was activated. The system was ready for use within a few minutes, when stable at liquid nitrogen temperature.

3. Transferring the EM grid. The cryo-grid storage box and the cassette in the transfer box were placed into position in the working space of the cryo-stage. EM 
grids were generally stored in liquid nitrogen after vitrification. The grids were transferred from the storage box to the cassette in the transfer box, which was subsequently transferred with the magnetic transfer tool to the cooled bridge for imaging (Fig. 10.2D).

4. Mounting the cryo-stage. The cryo-stage was transferred onto the light microscope, fastened onto the microscope stage with the screws, and the desired lens was selected. The stage was elevated to focus the grid.

5. Cryo-LM imaging. The appropriate filter was selected for scanning the grid in fluorescent mode for interesting positions. For targeting spots with excessive lipids, the fluorescent signal of FM5-95 was used. For targeting positions that were devoid of DNA, DAPI was used. Bright field images were always recorded alongside the fluorescent images. This image was necessary as an intermediate for correlating FM with EM images. Targeted positions were recorded preferentially near the center of the grid to improve relocation in TEM, because of the limited stage movement $(2 \times 2 \mathrm{~mm})$ and potential off-center positioning of the grid in the TEM. Images were named and stored such that it enabled easy tracking of the positions on the grid later in the TEM and when making the overlays.

6. Transferring grids and data. After imaging, the stage was dismounted from the microscope, the cassette transferred back into the cryo-grid box, and the box placed in a liquid-nitrogen-cooled vessel for transfer to the TEM. Recorded images were copied on to a laptop to make overlay images and to relocate the imaged positions in the TEM.

\subsubsection{IMAGE OVERLAY}

The cryo-LM images were used for three purposes: (i) relocation in TEM, (ii) annotation of recorded cryo-ET positions, and (iii) as basis for overlaying the cryo-LM, cryo-EM, and surface-rendered data. For relocation in the TEM, the cryo-LM images were first aligned to each other and overlaid in an Adobe Photoshop file, using layers.

1. Loading the images into Photoshop. The cryo-LM images that were recorded with different optical filters were imported into separate layers in Photoshop: File-Scripts-Load files into stack-Browse... and select images.

2. Aligning the layers. The layers could be automatically aligned during import into Photoshop by checking the box before "Attempt to Automatically Align source files" in the previous step. Dependent on the image content, this might not result in correctly aligned images. If the alignment did not work, a single layer was selected and aligned manually. Correct alignment of the layers was compared either by changing the opacity of the top layer or by alternating the visibility of the layer.

3. Coloring the images. If the fluorescent images were not colored, colors were assigned according to the fluorescent emission wavelength (e.g., red, green, or blue). The desired layer was selected (Window-Layer) and all other layers were 
made invisible. The channel panel was opened (Window-Channel) and the green and blue channels were shift-selected for a red color, red and blue channels for a green color, and red and green channels for a blue color. The selected layers were filled with black (Edit-Fill or Shift+F5) using the options: Contents: Use: Black; Blending Mode: Normal; Opacity: $100 \%$.

4. Adjusting brightness and contrast. To optimize visualization, the brightness and contrast of all layers were adjusted. This was done in a reversible way using adjustment layers. The desired layer was selected (Window-Layer), and selected layer and all other layers were made invisible. An adjustment layer was inserted (Layer-New adjustment layer-Brightness/Contrast) using the options: Color: none; Mode: Normal; Opacity 100\%. The adjustment layer appeared above the selected layer and acted on the first visible layer beneath it. To keep the adjustment layer acting on the desired cryo-LM image, there were several options. Both layers were linked: select both layers and select Link layers; or both layers were grouped: select both layers and Make new group from layers. "Linear dodge (Add)" was selected to overlay different color on a lowmagnification LM or EM background. The brightness/contrast icon in the adjustment layer (in the properties tab) was selected, legacy mode was selected, and brightness and contrast were changed using the sliders.

5. Save the image. The layered image was saved as a Photoshop document or layered tiff document. As filename the increasing CLEM experiment number were used, including, e.g., CLEM-1401.

The aligned cryo-LM images were transferred to a laptop that was able to open the layered images at the TEM. The transparent LM images were used to navigate to the correct grid square using the overall morphology of the mycelium. The fluorescence LM images and additional visible features on the EM grid such as grid bars, holes in the support film, and ice contamination were used to navigate to the desired spot within the mesh.

\subsubsection{CRYO-EM}

Cryo-EM was performed on a Titan Krios electron microscope (FEI, Eindhoven, The Netherlands). The use of the Titan Krios had several advantages for cryoCLEM on Streptomyces. First, the use of $300 \mathrm{keV}$ electrons in combination with zero-loss energy filtering enabled cryo-ET of the 500-800 nm thick Streptomyces. Higher-energy electrons have longer mean-free paths (the average distance between two scattering events), which reduced inelastic scattering. Zero-loss energy filtered imaging additionally filtered out the inelastically scattered electrons, thereby increasing the signal-to-noise ratio in the acquired images. This was especially important while imaging thick samples using tomography, since tilting increases the effective specimen thickness (twofold at $60^{\circ}$ ). Furthermore, the Titan Krios TEM system allowed high-throughput data collection of cryo-electron tomograms and enabled imaging of many positions on one (or multiple) EM grid(s). Effectively, this increased the yield of data collection 5- to 10-fold compared to imaging on 
earlier types of microscopes (e.g., Tecnai F20) that have side entry holder for only one grid.

1. EM at lowest magnification. The EM grid of choice was loaded into the electron microscope and visualized in the electron microscope at low magnification (typically $20 \times$ to $100 \times$ ). The beam and image shifts between LM, M, and SA magnification modes on the TEM were accurately aligned to keep the same feature centered in all magnifications.

2. Showing the cryo-LM images. The aligned cryo-LM image from the grid that was present in the TEM was opened using Photoshop on a laptop and the bright field image was displayed.

3. Relocating the grid square. Grid squares matching the LM imaging positions were relocated by eye and the stage positions were stored. When finder grids were used, the letters served as navigation. When nonfinder grids were used, relocation was guided by visual comparison of the Streptomyces morphology and the arrangement of holes in the carbon film. Sometimes the TEM projections and LM image were mirrored because the grid was flipped. This was corrected by flipping the LM image (Photoshop: Image-Image Rotation-Flip Canvas Horizontall Vertical).

4. Defining tomogram positions. The stored grid square positions were revisited and imaged at low magnifications, typically at $560 \times$ (comprising one complete grid square) and $2250 \times$ (for accurate determination of tomogram positions). Stage positions for tomograms were stored and annotated in the cryo-LM Photoshop documents. After tomographic data collection, additional projection images at $8700 \times$ or $15,000 \times$ were recorded for visualization and correlation of LM and EM data.

\subsubsection{CRYO-ELECTRON TOMOGRAPHY}

Tilt series were recorded using Xplore3D software (FEI Company) using the lowdose system and batch tomography. The stored positions for recording tomogram were revisited during set up of the batch tomography. Images were recorded with a $2 \mathrm{k} \times 2 \mathrm{k}$ CCD camera mounted on a postcolumn energy filter (Gatan Quantum SE 963, Gatan GmbH, Germany) in zero-loss mode using a slit width of 20 or $30 \mathrm{eV}$. Tomographic tilt series were recorded in two halves starting from $0^{\circ}$ to respective negative and positive maximum tilt angles. A linear tilt scheme was used with tilt angle increments of $3^{\circ}$ or $2^{\circ}$ and maximum angles of $51^{\circ}$ or $60^{\circ}$, respectively. Depending on the chosen tilt angle increment, images were recorded with an exposure time of 0.25 or $0.5 \mathrm{~s}$. The total electron dose that was used to acquire the full tilt series was in the order of $200-300 \mathrm{e} / \AA^{2}$. This dose was experimentally determined and kept the final images below the point of visual deterioration. The exposure time was increasing linearly with a factor of 1.6 between $0^{\circ}$ and $60^{\circ}$, while the dose was distributed over the whole series. Magnifications varied between $8.700 \times$ and $11,500 \times$, resulting in pixel sizes between 1.8 and $1.4 \mathrm{~nm}$. The defocus ranged between -15 and $-20 \mu \mathrm{m}$. Specimen shift correction during data collection 
was measured by the "tracking after" option on filtered images (long wavelength cutoff at $100 \pm 3 \mathrm{~nm}$ and $2 \pm 0.5 \mathrm{~nm}$ low wavelength cutoff, with a taper filter of 8 pixels and the Sobel filter and remove X-ray options on). At the highest tilt angles, defocus was checked every two tilt angles, while at the lower tilt angles, defocus was not checked. Total acquisition time ranged between 20 and $55 \mathrm{~min}$, depending on acquisition parameters; in an overnight, batch up to 35 tomograms could be recorded.

\subsubsection{TOMOGRAPHIC RECONSTRUCTION}

Image processing was performed using the IMOD software suite (Kremer, Mastronarde, \& McIntosh, 1996). Here, we briefly describe the steps taken and parameters chosen for the reconstruction of cryo-electron tomograms of Streptomyces taken on the Titan Krios described above. A more detailed description on tomographic reconstruction can be found elsewhere (Koning \& Koster, 2013).

1. Preparing tilt series. The recorded electron tomography tilt series and accompanying files were copied to a separate folder. The .mrc extension of the tilt series was renamed to .st.

2. Opening tilt series. Etomo was started and the tilt series was opened. "Single axis" and "Single frame" were selected in the "Data type." Next, "Scan header" was selected and the value for the diameter of the used fiducials was added in the appropriate box. "View Raw Image Stack" was selected and the numbers of bad images (e.g., images with partly blocked beam, blurred images, low contrast images, or badly focused images) were selected in the "Exclude views" box. "Create Com Scripts" was clicked.

3. Preprocessing tomogram. Hot pixels were removed via one detection round of CCD eraser function using default settings: Peak criterion: 10; Difference criterion: 8; Maximum radius: 2.6. If high-intensity streaks were present in the images, several rounds of the CCD eraser were used or the parameters were changed to fully remove the hot pixels.

4. Prealigning tilt series. A first, course alignment of the tilt series was performed by cross-correlation using the default parameters. In some cases, the course alignment did not work correctly, e.g., because parts of the images were obscured by thick ice, resulting in abrupt image shifts. In this case, the alignment was improved by changing the default values of the cross-correlation parameters, by trimming pixels, or by manual alignment of the tilt series using MIDAS.

5. Generation fiducial model. Gold fiducials of $15 \mathrm{~nm}$ were used for the fine alignment of the tilt series using the "Make seed and track option." In our case, patch tracking was less efficient because of limited contrast in the tilt series images of the Streptomyces. Up to 10 fiducials positioned as close as possible to the aligned object were initially selected and tracked along the whole tilt series. In subsequent rounds, more fiducials were added. Alternatively, the RAPTOR option within IMOD was used to automatically generate an initial fiducial model. 
6. Fine align tilt series. Fine alignment was performed starting with the following parameters: "Threshold for residual report" 3; "Relative to all neighbouring views"; "Do not sort fiducials into 2 surfaces"; "Enable local alignments" off; "Solve for all rotations"; "Fixed magnification at 1.0"; "Group tilt angles"; "Distortion Solution type" disabled. Tracked beads with big residuals were manually centered to the beads. Bead positions in low signal-to-noise positions and aggregated beads were removed. After one or two rounds of refinement, residual report was decreased in steps to 1 , and "Solve for all magnifications" and "Solve for all except minimum" tilt angles were activated. "Final global mean residual" was below 1 .

7. Positioning tomogram. Since the specimens were thick whole Streptomyces cells (and not sections), "Create boundary model" was not critical or necessary to minimize the reconstructed volume or straighten the sample in the volume. "Sample tomogram thickness" was set to 1200; "Use whole tomogram" and "Binning" 3 were used to "Create a whole tomogram" and determine the thickness of the final tomogram.

8. Fine align stack. Fine alignment of the stack was done using "Binning" 1 and visually inspected for quality. "CTF correction," "Gold erasing," and "2D filtering" were not usually performed.

9. Generating tomogram. Tomograms were generated using "Back Projection" using default values. Alternatively, SIRT reconstructions were calculated using 5-10 iterations. SIRT reconstructions generated tomograms with more contrast for large features.

10. Postprocess tomogram. The tomograms were postprocessed by "Swap y and $\mathrm{z}$ dimensions"; "Conversion to bytes" and standard values were used for "Scaling from sections." To reduce the tomogram file size for loading and rendering in Amira, sometimes volume trimming and "Squeeze vol" (using an isotropic reduction factor of 2) were performed.

11. Filter tomogram. Noise reduction and contrast enhancement of (weighted backprojected) tomograms were performed using nonlinear anisotropic diffusion in etomo (File-New Nonlinear Anisotropic Diffusion). To determine suitable parameter settings for this algorithm, a typical single $\mathrm{Z}$ slice was selected from the file and the effect of $\mathrm{k}$ values and iterations were evaluated for contrast enhancement by visual inspection. Typical values used were between 20 and 200 for $\mathrm{k}$ and between 10 and 20 for the number of iterations.

\subsubsection{SEGMENTATION AND VOLUME RENDERING}

The main objective for performing segmentation and surface rendering was to extract and visualize specific structures inside tomograms. Surface rendering provides better impression of structures in 3D and makes the information in a tomogram accessible for a broader audience. Segmentation, surface rendering, and three-dimensional visualization were performed using Amira. Segmentation was performed by rough manual masking of structures, followed by fine-tuning by histogram thresholding 
of highest density voxels and subsequent smoothing by a combination of small islands removal and iterative growing/shrinking of selected voxels (erosion/dilation). Image segmentation was performed on nonlinear anisotropic diffusion-filtered or SIRT-reconstructed datasets only. Nevertheless, manual segmentation was necessary because of the high noise levels in the tomograms.

1. Loading tomogram. The tomogram was loaded into AMIRA using "File-open data (or Ctrl+O)" or "Open data..." in the pool. An orthoslice was attached to the data: select data and select "Orthoslice" in the pool (or right-click data and select orthoslice). In the properties window, the orientation of the slice, the contrast, and the slice number could be varied.

2. Rough segmentation. Specific structures of interest were manually selected using the AMIRA segmentation editor. In the editor, a new label was created, and new materials were created for each structure to be segmented. First, the complete Streptomyces cell was outlined and selected by interpolating several circles or ovals that were positioned along the length of the cell in end-on views every 10 th to 50th slice using the brush tool. Other structures, like the cytoplasm or vesicles, were segmented in a similar fashion and added to the separate materials. Note that in the data area, the visibility in 2D slices and 3D volume can be switched on and off and a material can be selected or locked, to prevent further changes.

3. Fine segmentation. Fine segmentation was performed by selecting the voxels with highest density from the roughly segmented structures using a histogram. In "Display and Masking," the "Masking: enable" and "Masking: show" buttons were ticked. Selected voxels became marked in purple. "Threshold" with options "All slices" and "Current Material Only" on was selected. The sliders of the histogram window were changed such that lowest values up to the desired gray value were included. The existing material with the current selection was replaced.

4. Smoothing selection. The segmented voxels after histogram selection made up a noisy and discontinuous selection that did not result in a usable surface rendering. The segmentation was improved by removing small islands ("SegmentationRemove Islands" using "Mode: 3D volume" increasing the size from 15 to a desired value until most small islands are removed), smoothing of labels ("Segmentation-Smooth labels" selecting "Mode: 3D volume" and size 3 and using several rounds of smoothing to get desired effect), and subsequent growing and shrinking of the selection in 3D.

5. Make surface rendering. A surface-rendered view of the segmented data was visualized in the object pool. (A "SurfaceGen" module was applied to the selected data object ending with ".labels." A surface view module was applied to the data object ending with ".surf" to surface render the segmented volume.) Note that different materials could be switched on and off and surfaces could be made transparent. For a mix of transparent and solid views of different materials, a duplicate of the "SurfaceView" was necessary. 
6. Make animation. The surface-rendered objects were used to produce an animation that was later integrated with the LM and 2D EM data. Only a short overview of the procedure is given here since the choice for animations varies with the dataset and individual preferences. Movie sequences were made using the "DemoMaker" module ("Create-Animation/Demo-DemoMaker" and select "DemoDirector" to visualize the timeline). Multiple consecutive events were added to make the complete movie ("New event. .." to add the first sequence. For example, for making a tomographic slice sequence, expand "Orthoslice" and select "Slice Number." In the "Properties Window," select the start and end values of both slice number and time). Rotating objects were created using "Create-Animation/Demo-Object rotate." Once the object rotate module was activated, it could be selected in the "DemoMaker" module. The "MovieMaker" module was used to make a movie from the sequence.

\subsubsection{VISUALIZATION OF CORRELATIVE DATA}

Our goal for the visualization was to convincingly correlate the LM, EM, and surface-rendered tomographic data into a continuous sequence. In our experience, before starting to visualize the correlation, it was convenient to group all data necessary for a single correlation into a single computer folder assigned with a CLEM experiment number. One CLEM experiment typically contains cryo-LM data from one grid square position and cryo-EM 2D overview images from the square, multiple higher-magnification cryo-EM images with accompanying cryo-electron tomograms and surface-rendered tomograms.

1. Opening the overlay image. The previously made file containing the aligned LM channels in a layered .psd file was opened in Adobe Photoshop.

2. Adding EM overview image. In order to place the complete EM overview image onto the LM image, the canvas size was increased by $200 \%$. The lowmagnification EM overview of a grid square was placed in a separate layer ("File-place"). This layer was made partly transparent (Set layer opacity to 50\%) in order to view the EM and LM images simultaneously. The EM overview image was mirrored (if necessary), shifted, and rotated ("Edit-transform" or "Shift + T") such that all visible features (holey carbon and Streptomyces) optimally fitted the transparent LM and FM images.

3. Adding high-magnification EM images. The image size was increased ("Edit-Image size" (or alt + ctrl $+\mathrm{I}$ ), selecting "Percent" in "Pixel dimensions" or "Document size" and setting these to $200 \%, 400 \%$, or $800 \%$ ) to place highermagnification overview images without resolution loss. In order to prevent excessive document file size, unnecessary parts of the LM image were cropped. The medium-magnification EM overview were placed in a separate layer and fitted as was described above. For correct scaling and placement, the pixel size and microscope coordinates of the images were used. Tomographic slices were added in the same way as done for high-magnification images. Alignment of the tomographic slices over high-magnification EM images was aided by the 
edges of holes in the carbon and fiducial gold markers. The resulting overlay .psd file served as basis for a movie including the surface-rendered data that can be made with Adobe Premiere.

4. Making movies of correlative data. Movie sequences were produced using Adobe Premiere. All correlative data were integrated to produce a movie that zoomed in from large overview LM images to the high-resolution EM and surface-rendered data. The image size of the Photoshop file was reduced to $10-25 \%$ of the original before they were imported into Adobe Premiere, because images were much larger than the resolution of HD movies. The Photoshop document was imported into a new Premiere project, retaining the layers and alignments ("File-Import," select Import as "Individual layers"). A new sequence was opened and the images were dragged into different video layers in the sequence. The image scale was adapted to fit the resolution of the project (Select image and in video effects "Motion-Scale" to $10 \%$ ). Movies were produced from the correlative data by seamlessly zooming in from the LM images via the overview EM images, to the high-magnification EM images, by changing the scaling and transparencies. In the end, the 3D tomographic and surface-rendered data that were made in Amira were seamlessly integrated.

\subsection{MATERIALS AND INSTRUMENTATION}

a. Cryo- $L M$

- Linkam CMS196 cryo-stage (Linkam, UK).

- Leitz DMRB upright light microscope (Leica, Wetzlar, Germany) with an adapted microscope stage to hold the CMS196 and an adapted condenser lens (Linkam) for transparent light imaging. The microscope was equipped with a Hi Plan $10 \times$ objective lens with a NA of 0.22 and a HCXPL Fluortar $100 \times$ dry objective lens with an NA of 0.71 and a working distance of $4.7 \mathrm{~mm}$. The microscope was equipped with a Leica TRITC filter with 515-560 nm excitation and $590 \mathrm{~nm}$ long-pass detection filter, a Leica UVA filter with excitation from 340 to $380 \mathrm{~nm}$, and a $425 \mathrm{~nm}$ long-pass detection filter. Images were recorded using a $100 \times$ dry objective with a working distance of $4.7 \mathrm{~mm}$ and a NA of 0.75 . Images were recorded with a Leica DFC350FX CCD camera using Leica Application Suite Advanced Fluorescence Lite software (LAS-AF-Lite 2.6.0, Leica, Austria).

b. Cryo-EM

- Gatan Digital Micrograph (Gatan, München, Germany).

- Xplore3D, version 4 (FEI Company, Eindhoven, The Netherlands).

- Titan Krios TEM (Krios 1, NeCEN) (FEI, Eindhoven, The Netherlands), equipped with Quantum SE/963 energy filter (Gatan, München, Germany).

c. Image processing

- IMOD version 4.5 (Boulder, CO, USA).

- Amira version 5.4 (FEI Company, Eindhoven, The Netherlands).

- Photoshop CS6 and Premiere CS6 (Adobe, CA, USA). 


\subsection{DISCUSSION AND OUTLOOK}

The presented method describes a two-step cryo-CLEM approach in which vitrified samples are imaged using cryo-fluorescence LM, followed by cryo-electron tomography. The acquired images and reconstructed data, including transparent and fluorescence LM, EM projection images, three-dimensional electron tomograms, and surface-rendered data, have different scales, dimensions, and modalities and are correlated and integrated into overlay images (Fig. 10.3) and a continuously scaling image sequence.
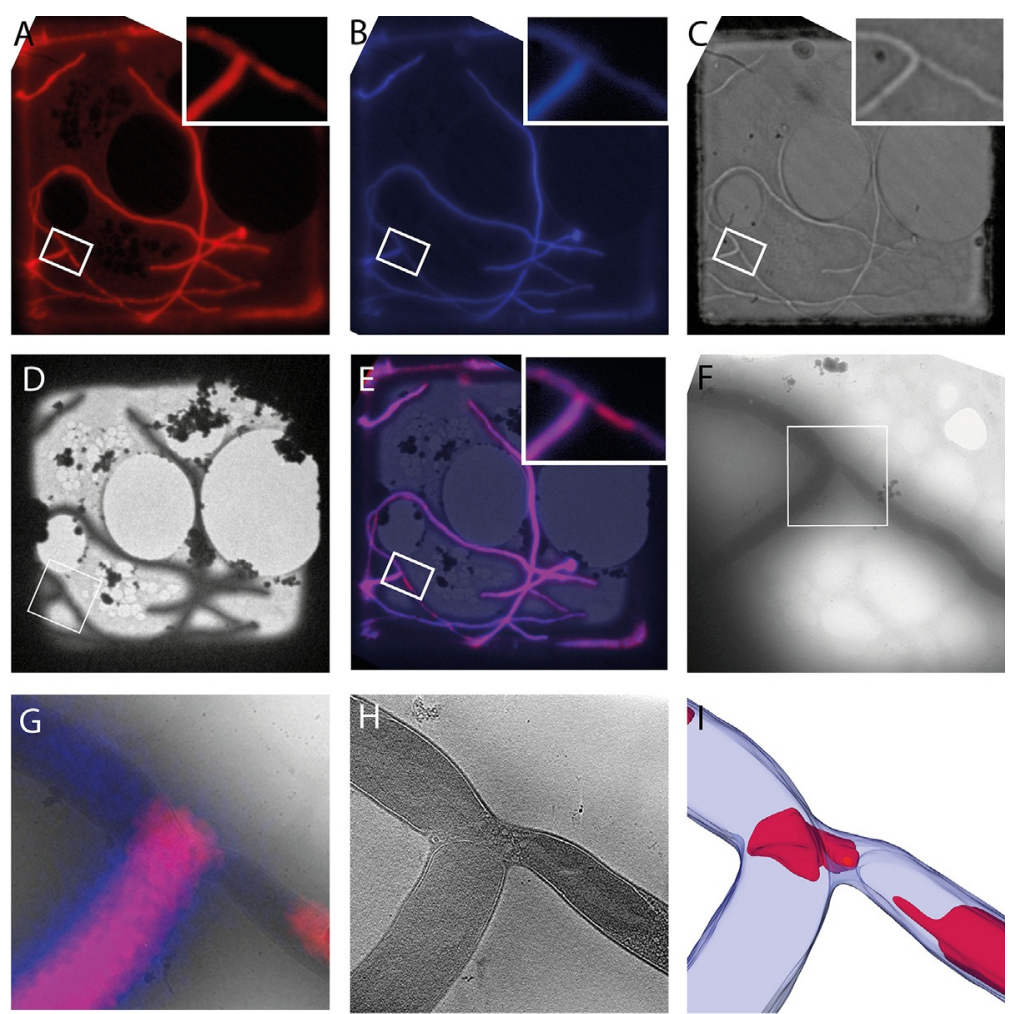

\section{FIGURE 10.3}

Cryo-CLEM of Streptomyces. The cryo-fluorescence microscopy using FM5-95 stain for membranes (A) and DAPI stain for DNA in (B) are used to determine target positions for cryo-ET. The insets in $A$ and $B$ show (denoted by the white rectangle in $A, B, C$, and $E$ ) a region of interest with membrane and DNA staining. The cryo-bright field microscopy image (C) and the cryo-electron microscopy projection overview (D) are used to aid correlation of the fluorescent images ( $A$ and $B$ ) with higher-magnification cryo-EM images $(F, G)$ and electron tomogram slices $(\mathrm{H})$ and surface-rendered tomogram (I, cell wall in blue and membranes in red). 
This two-step cryo-CLEM approach, using a cryo-light microscopic stage in combination with a cryo-TEM, has been applied before, using different cryo-stages and TEMs (Carlson \& Evans, 2011; Sartori et al., 2007; Schwartz et al., 2007; van Driel et al., 2009). The currently used setup with the improved specimen handling and transfer capabilities of the Linkam CMS196 freezing stage in combination with automated data collection using a FEI Titan Krios electron microscope was advantageous compared to our earlier experiments (van Driel et al., 2009). It improved the handling of cryogenically cooled specimens, the quality of specimens, the success rate of the experiments, and the amount of cryo-electron tomograms, resulting in tens of useful correlations within a few weeks of data acquisition.

The use of Streptomyces for the development of the here described cryo-CLEM method had several advantages. In earlier cryo-CLEM investigations, subcellular structures-microtubules, mitochondria, or viruses-were targeted in eukaryotic cells (Gruska, Medalia, Baumeister, \& Leis, 2008; Jun et al., 2011; Sartori et al., 2007; Schwartz et al., 2007; van Driel et al., 2009). Finder grids were employed to navigate to the correct EM grid square, while visual clues were used for targeting specific subcellular structures. Recently, the addition of electron dense and fluorescent beads to the specimen was shown to enable high-precision alignment of LM and EM images (Fukuda et al., 2014; Schellenberger et al., 2014; Schorb \& Briggs, 2014). Streptomyces are multicellular bacteria which grow in the form of a branching mycelium. When cryo-fixed on an EM grid, their distinct pattern allowed visual relocation of targets from the light to the electron microscope and reasonably accurate overlay of LM and EM images based solely on the Streptomyces hyphal morphology, without the use of finder grids.

One major disadvantage of the two-step cryo-CLEM method is that the extra cryo-LM imaging step increases the amount of handling and transfers of cryogenically cooled specimens needed as opposed to cryo-EM on its own. These vitrified EM grids are very sensitive to handling and ice contamination from humid air and accidental temperature fluctuations. Any added handling step decreases the success rate of an experiment. A solution is the recently developed integrated LM and EM systems (Faas et al., 2013; Iijima et al., 2014; www.fei.com/correlativemicroscopy), in which no additional transfer step is necessary since LM is performed within the TEM. Additionally, these integrated systems allow fast and easy relocation of targeted structures and enable automatic overlay of 2D LM and EM images. Disadvantages are that the LM and EM microscope types are fixed, the resolution of the LM data in current light and electron microscopes is compromised by the relatively low NA of the lens, and the TEM extraction voltage $(80-120 \mathrm{keV})$ is not optimal for cryo-ET on thick cells. In fact, most parts of any whole cell are too thick for cryo-ET imaging without being thinned to less than $300 \mathrm{~nm}$. Cryo-sectioning of cells (Al Amoudi et al., 2004) and cryo-FIB methods (Marko, Hsieh, Moberlychan, Mannella, \& Frank, 2006; Rigort et al., 2010) can be used to thin cells for imaging by cryo-ET, and these methods are being explored for integration with cryo-CLEM methods (Faas et al., 2013; Fukuda et al., 2014). 
An important additional development in the field of CLEM is combining LM of live cells with EM, adding the temporal component of tagged structures to the static high-resolution cellular morphology. Currently used CLEM methods combine live imaging with subsequent fixation of live cells, either chemically (Mironov, Polishchuk, \& Beznoussenko, 2008), high-pressure freezing (Verkade, 2008), or plunge freezing (Jun et al., 2011) with electron tomographic imaging. The recent integration of a light microscope into a plunge-freezing device reduces the amount of additional specimen transfer steps and sets the time resolution between live imaging and vitrification to a few seconds (Koning et al., 2014).

A key future improvement for cryo-CLEM applications is to bridge the resolution gap between LM (few hundred nanometers) and cryo-TEM (few nanometers) to improve the localization accuracy of fluorescently tagged structures. The current localization accuracy using markers is in the order of tens of nanometers (Schellenberger et al., 2014; Schorb \& Briggs, 2014), which is still one order larger than the resolution that can be achieved by cryo-ET. The use of super-resolution LM techniques might enable precise localization of individual GFP-tagged proteins.

With respect to the visual integration of data, a single platform for visualizing LM and EM projection images with live imaging sequences, electron tomography, and surface-rendered data is desirable. While many software programs perform well in correlating LM and EM data (Adobe Photoshop), visualizing different types of data (Amira), and making movie sequences from these (Adobe Premiere), we have found no program that is tailored to performing both the correlation and the visualization of multidimensional data which varies several orders in size.

We think that the described cryo-CLEM methods that we developed here for Streptomyces are applicable with only minor adaptions-e.g., using reference grids for relocation, markers for correlation, different fluorescent labels, different freezing stages, and electron microscopes-for many other biological specimens.

\section{ACKNOWLEDGMENTS}

We would like to thank Marjon Mourik for critical reading of the manuscript. This research has been executed with support of Cyttron II (LSH framework FES0908), NeCEN, The Netherlands Centre for Electron Nanoscopy, Leiden, The Netherlands, and NWO, The Netherlands Organisation for Scientific Research and is partly financed by the European Regional Development Fund of the European Commission.

\section{REFERENCES}

Al Amoudi, A., Chang, J. J., Leforestier, A., McDowall, A., Salamin, L. M., Norlen, L. P., et al. (2004). Cryo-electron microscopy of vitreous sections. The EMBO Journal, 18, 3583-3588.

Bibb, M. J. (2005). Regulation of secondary metabolism in streptomycetes. Current Opinion in Microbiology, 2, 208-215. 
Carlson, D. B., \& Evans, J. E. (2011). Low-cost cryo-light microscopy stage fabrication for correlated light/electron microscopy. Journal of Visualized Experiments, (52).

Celler, K., Picioreanu, C., van Loosdrecht, M. C., \& van Wezel, G. P. (2012). Structured morphological modeling as a framework for rational strain design of Streptomyces species. Antonie van Leeuwenhoek, 3, 409-423.

Chater, K. F., \& Losick, R. (1997). Mycelial life style of Streptomyces coelicolor A3(2) and it's relatives. Oxford University Press, 149-182.

Claessen, D., Rozen, D. E., Kuipers, O. P., Sogaard-Andersen, L., \& van Wezel, G. P. (2014). Bacterial solutions to multicellularity: A tale of biofilms, filaments and fruiting bodies. Nature Reviews. Microbiology, 2, 115-124.

Diestra, E., Fontana, J., Guichard, P., Marco, S., \& Risco, C. (2009). Visualization of proteins in intact cells with a clonable tag for electron microscopy. Journal of Structural Biology, 3, $157-168$.

Dubochet, J., Adrian, M., Chang, J. J., Homo, J. C., Lepault, J., McDowall, A. W., et al. (1988). Cryo-electron microscopy of vitrified specimens. Quarterly Reviews of Biophysics, 2, 129-228.

Errington, J., Daniel, R. A., \& Scheffers, D. J. (2003). Cytokinesis in bacteria. Microbiology and Molecular Biology Reviews, 1, 52-65, table of contents.

Faas, F. G., Avramut, M. C., van den Berg, B. M., Mommaas, A. M., Koster, A. J., \& Ravelli, R. B. (2012). Virtual nanoscopy: Generation of ultra-large high resolution electron microscopy maps. The Journal of Cell Biology, 3, 457-469.

Faas, F., Bárcena, M., Agronskaia, A., Gerritsen, H., Moscicka, K., Diebolder, C., et al. (2013). Localization of fluorescently labeled structures in frozen-hydrated samples using integrated light electron microscopy. Journal of Structural Biology, 3, 283-290.

Fukuda, Y., Schrod, N., Schaffer, M., Feng, L. R., Baumeister, W., \& Lucic, V. (2014). Coordinate transformation based cryo-correlative methods for electron tomography and focused ion beam milling. Ultramicroscopy, 143, 15-23.

Gruska, M., Medalia, O., Baumeister, W., \& Leis, A. (2008). Electron tomography of vitreous sections from cultured mammalian cells. Journal of Structural Biology, 3, 384-392.

Iancu, C. V., Tivol, W. F., Schooler, J. B., Dias, D. P., Henderson, G. P., Murphy, G. E., et al. (2006). Electron cryotomography sample preparation using the Vitrobot. Nature Protocols, 6, 2813-2819.

Iijima, H., Fukuda, Y., Arai, Y., Terakawa, S., Yamamoto, N., \& Nagayama, K. (2014). Hybrid fluorescence and electron cryo-microscopy for simultaneous electron and photon imaging. Journal of Structural Biology, 1, 107-115.

Jakimowicz, D., \& van Wezel, G. P. (2012). Cell division and DNA segregation in Streptomyces: How to build a septum in the middle of nowhere? Molecular Microbiology, 3, 393-404.

Jun, S., Ke, D., Debiec, K., Zhao, G., Meng, X., Ambrose, Z., et al. (2011). Direct visualization of HIV-1 with correlative live-cell microscopy and cryo-electron tomography. Structure, $11,1573-1581$.

Karreman, M. A., Agronskaia, A. V., van Donselaar, E. G., Vocking, K., Fereidouni, F., Humbel, B. M., et al. (2012). Optimizing immuno-labeling for correlative fluorescence and electron microscopy on a single specimen. Journal of Structural Biology, 2, 382-386.

Kieser, T., Bibb, M. J., Buttner, M. J., Chater, K. F., \& Hopwood, D. A. (2000). Practical Streptomyces genetics. U.K., John Innes Foundation: Norwich.

Koning, R. I. (2010). Cryo-electron tomography of cellular microtubules. Methods in Cell Biology, 97, 455-473. 


\section{CHAPTER 10 cryo-CLEM of Streptomyces}

Koning, R. I., Faas, F. G., Boonekamp, M., de Visser, B., Janse, J., Wiegant, J. C., et al. (2014). MAVIS: An integrated system for live microscopy and vitrification. Ultramicroscopy, 143, 67-76.

Koning, R. I., \& Koster, A. J. (2013). Cellular nanoimaging by cryo electron tomography. Methods in Molecular Biology, 950, 227-251.

Kremer, J. R., Mastronarde, D. N., \& McIntosh, J. R. (1996). Computer visualization of threedimensional image data using IMOD. Journal of Structural Biology, 1, 71-76.

Kukulski, W., Schorb, M., Welsch, S., Picco, A., Kaksonen, M., \& Briggs, J. A. (2011). Correlated fluorescence and 3D electron microscopy with high sensitivity and spatial precision. The Journal of Cell Biology, 1, 111-119.

Le Gros, M. A., McDermott, G., Uchida, M., Knoechel, C. G., \& Larabell, C. A. (2009). Highaperture cryogenic light microscopy. Journal of Microscopy, 1, 1-8.

Lucic, V., Leis, A., \& Baumeister, W. (2008). Cryo-electron tomography of cells: Connecting structure and function. Histochemistry and Cell Biology, 2, 185-196.

Marko, M., Hsieh, C., Moberlychan, W., Mannella, C. A., \& Frank, J. (2006). Focused ion beam milling of vitreous water: Prospects for an alternative to cryo-ultramicrotomy of frozen-hydrated biological samples. Journal of Microscopy, 222(Pt. 1), 42-47.

Marko, M., Hsieh, C., Schalek, R., Frank, J., \& Mannella, C. (2007). Focused-ion-beam thinning of frozen-hydrated biological specimens for cryo-electron microscopy. Nature Methods, 3, 215-217.

McCormick, J. R. (2009). Cell division is dispensable but not irrelevant in Streptomyces. Current Opinion in Microbiology, 6, 689-698.

Mironov, A. A., Polishchuk, R. S., \& Beznoussenko, G. V. (2008). Combined video fluorescence and 3D electron microscopy. Methods in Cell Biology, 88, 83-95.

Mistry, B. V., Del Sol, R., Wright, C., Findlay, K., \& Dyson, P. (2008). FtsW is a dispensable cell division protein required for Z-ring stabilization during sporulation septation in Streptomyces coelicolor. Journal of Bacteriology, 16, 5555-5566.

Morris, D. M., \& Jensen, G. J. (2008). Toward a biomechanical understanding of whole bacterial cells. Annual Review of Biochemistry, 77, 583-613.

Pilhofer, M., Ladinsky, M. S., McDowall, A. W., \& Jensen, G. J. (2010). Bacterial TEM: New insights from cryo-microscopy. Methods in Cell Biology, 96, 21-45.

Resch, G. P., Brandstetter, M., Königsmaier, L., Urban, E., \& Pickl-Herk, A. M. (2011). Immersion freezing of suspended particles and cells for cryo-electron microscopy. Cold Spring Harbor Protocols, 7, 803-814.

Rigort, A., Bauerlein, F. J., Leis, A., Gruska, M., Hoffmann, C., Laugks, T., et al. (2010). Micromachining tools and correlative approaches for cellular cryo-electron tomography. Journal of Structural Biology, 2, 169-179.

Risco, C., Sanmartin-Conesa, E., Tzeng, W. P., Frey, T. K., Seybold, V., \& de Groot, R. J. (2012). Specific, sensitive, high-resolution detection of protein molecules in eukaryotic cells using metal-tagging transmission electron microscopy. Structure, 5, 759-766.

Sartori, A., Gatz, R., Beck, F., Rigort, A., Baumeister, W., \& Plitzko, J. M. (2007). Correlative microscopy: Bridging the gap between fluorescence light microscopy and cryo-electron tomography. Journal of Structural Biology, 2, 135-145.

Schellenberger, P., Kaufmann, R., Siebert, C. A., Hagen, C., Wodrich, H., \& Grunewald, K. (2014). High-precision correlative fluorescence and electron cryo microscopy using two independent alignment markers. Ultramicroscopy, 143, 41-51. 
Schorb, M., \& Briggs, J. A. (2014). Correlated cryo-fluorescence and cryo-electron microscopy with high spatial precision and improved sensitivity. Ultramicroscopy, 143, 24-32.

Schwartz, C. L., Sarbash, V. I., Ataullakhanov, F. I., Mcintosh, J. R., \& Nicastro, D. (2007). Cryo-fluorescence microscopy facilitates correlations between light and cryo-electron microscopy and reduces the rate of photobleaching. Journal of Microscopy, 2, 98-109.

Schwedock, J., McCormick, J. R., Angert, E. R., Nodwell, J. R., \& Losick, R. (1997). Assembly of the cell division protein FtsZ into ladder-like structures in the aerial hyphae of Streptomyces coelicolor. Molecular Microbiology, 5, 847-858.

Tivol, W. F., Briegel, A., \& Jensen, G. J. (2008). An improved cryogen for plunge freezing. Microscopy and Microanalysis, 5, 375-379.

van Driel, L. F., Valentijn, J. A., Valentijn, K. M., Koning, R. I., \& Koster, A. J. (2009). Tools for correlative cryo-fluorescence microscopy and cryo-electron tomography applied to whole mitochondria in human endothelial cells. European Journal of Cell Biology, 11, 669-684.

van Wezel, G. P., \& McDowall, K. J. (2011). The regulation of the secondary metabolism of Streptomyces: New links and experimental advances. Natural Product Reports: A Journal of Current Developments in Bioorganic Chemistry, 7, 1311-1333.

van Wezel, G. P., McKenzie, N. L., \& Nodwell, J. R. (2009). Chapter 5. Applying the genetics of secondary metabolism in model actinomycetes to the discovery of new antibiotics. Methods in Enzymology, 458, 117-141.

Verkade, P. (2008). Moving EM: The Rapid Transfer System as a new tool for correlative light and electron microscopy and high throughput for high-pressure freezing. Journal of Microscopy, 230(Pt. 2), 317-328.

Wang, Q., Mercogliano, C. P., \& Lowe, J. (2011). A ferritin-based label for cellular electron cryotomography. Structure, 2, 147-154. 